\title{
GENERALIZED INTERSECTION MULTIPLICITIES OF MODULES. II
}

\author{
SANKAR P. DUTTA
}

\begin{abstract}
The vanishing conjecture for intersection multiplicities in dimensions $\leqslant 5$ is true in greater generality than previously known.
\end{abstract}

In this note we prove a more general theorem than Corollary 2.5 of [D1]. Since both the vanishing and nonvanishing conjectures are not true in general [DHM], it is now important to understand the following question: What can we say about

$$
\chi^{R}(M, N)=\sum_{i=0}^{d}(-1)^{i} l\left(\operatorname{Tor}_{i}^{R}(M, N)\right),
$$

where $R$ is a Gorenstein ring, and $M$ and $N$ are modules over $R$ with finite projective dimension such that $l\left(M \otimes_{R} N\right)<\infty, \operatorname{dim} M+\operatorname{dim} N \leqslant \operatorname{dim} R$, and $d=$ p.d. $M$ ?

The following theorems partially answer the question up to dimension 5 .

Throughout $\$ 1$ all tensor products, Tor's and Euler characteristics are computed over $R$.

1.1. TheOREM. Let $R$ be a Gorenstein ring of dimension $\leqslant 5$. Let $M$ and $N$ be modules of finite projective dimension with $l(M \otimes N)<\infty$ and $\operatorname{dim} M+\operatorname{dim} N<$ $\operatorname{dim} R$. Then $\chi(M, N)=0$.

PROOF. First we note that $\$ \S 1.1$ and 1.3 of [D1] hold true in the above case. The sufficient part of Theorem 2.4 of [D1] is still valid, though the statement has to be changed a bit in the following way:

1.2. THEOREM. Let $R$ be a Gorenstein ring of $\operatorname{dim} N$. If for any two perfect modules $M$ and $N$ with (i) $\operatorname{dim} M+\operatorname{dim} N=\operatorname{dim} R$, and (ii) $l(M \otimes N)<\infty, l(M \otimes N)=$ $l(M \otimes \check{N})$, then the vanishing conjecture holds in $R$ for every pair of modules of finite projective dimension. Here $\check{N}=\operatorname{Ext}^{n-r}(N, R), r=\operatorname{dim} N$.

Proof of this theorem can be obtained by following the argument of (2.4) of [D1].

To prove our theorem, by 1.2 it is enough to prove that

$$
l\left(T \otimes_{R} Q\right)=l\left(T \otimes_{R} \check{Q}\right) \ldots,
$$

where $T$ and $Q$ are any two perfect modules over $R$ with $l(T \otimes Q)<\infty$ and $\operatorname{dim} T+\operatorname{dim} Q=\operatorname{dim} R$.

Received by the editors August 29, 1983 and, in revised form, April 30, 1984.

1980 Mathematics Subject Classification. Primary 13H15; Secondary 13D99, 14C17. 
Case $1 . \operatorname{dim} T=5$, i.e., $T$ is free, hence $l(Q)<\infty$. Since $l(Q)=l(\check{Q}), l(T \otimes Q)=$ $l(T \otimes \check{Q})$.

Case 2. $\operatorname{dim} T=4$. In this case by killing an $R$-sequence which is also an $M$-sequence of length four contained in $\mathrm{Ann}_{R} Q$, we reduce the proof to proving (1) on a Gorenstein ring of dimension 1 and, hence, we are done.

Case 3. $\operatorname{dim} T=3$. In this case killing an $M$-sequence which is also an $R$-sequence of length 3, contained in $\mathrm{Ann}_{R} Q$, we reduce the proof to proving (1) on a Gorenstein ring of $\operatorname{dim} 2$ and we are done by (1.1) of [D1].

Similar arguments hold true when $\operatorname{dim} R \leqslant 4$.

The next theorem throws a little more light on the nonvanishing conjecture. (This is a changed version of Proposition (3.4) of [D1].)

1.3. THEOREM. Let $R$ be a complete Gorenstein ring of $\operatorname{dim} 5$ whose coefficient ring is a discrete valuation ring $V$, and let $p$ be a generator of the maximal ideal of $V$. Let $M$ and $N$ be two modules over $R$ such that (i) $M$ is perfect and p.d. $N<\infty$, (ii) $l\left(M \otimes_{R} N\right)<\infty$, (iii) $\operatorname{dim} M+\operatorname{dim} N=5$, (iv) $p N=0$, and $p$ is a n.z.d. on $M$. Then $\chi(M, N)>0$.

For the proof we refer the reader to (1.8) and Corollaries 5 and 6 of [D2].

REMARK. Theorems (2.2) and (2.4) of [D1] and Theorem 1.1 can be proved with the assumption that $R$ is a Cohen-Macaulay ring. The techniques are the same; one has only to consider spectral sequences $\operatorname{Ext}_{S}^{i}\left(\operatorname{Tor}_{j}^{R}(M, N), S\right)$ and $\operatorname{Ext}_{R}^{i}\left(M, \operatorname{Ext}_{S}^{j}(N, S)\right)$, and instead of taking $\check{M}, \check{N}$ as defined in the Gorenstein case with p.d. $M=d$, say, one should consider $\tilde{M}=\operatorname{Ext}_{R}^{d}(M, R)$ and $\check{N}=\operatorname{Ext}_{S}^{n-r}(N, S)$, $r=\operatorname{dim} N$. Here $S$ is a Gorenstein ring such that $R=S / I$ and $\operatorname{dim} R=\operatorname{dim} S$ (assuming $R$ to be complete).

\section{REFERENCES}

[C-E] H. Cartan and S. Eilenberg, Homological algebra, Chapter XVI, Princeton Univ. Press, Princeton, N. J., 1973.

[D-H-M] S. P. Dutta, M. Hochster and J. E. McLaughlin, Modules of finite projective dimension with negative intersection multiplicity, preprint.

[D1] , Generalized intersection multiplicities of modules, Trans. Amer. Math. Soc. 276 (1983), 657-669.

[D2] Frobenius and multiplicities, J. Algebra 85 (1983), 424-448.

[H] M. Hochster, Conference on commutative algebra, Lecture Notes in Math., vol. 311, Springer-Verlag, Berlin and New York, 1973, pp. 120-152.

Department of Mathematics, University of Pennsylvania, Philadelphia, Pennsylvania 19104 School of Mathematics, Institute for Advanced Study, Princeton, New Jersey 08540 